Original Research Article

\title{
Antidepressants-a possibly risk factor for cataract development: a cross-sectional study
}

\author{
Rajnish Raj ${ }^{1 *}$, Anuradha Raj' ${ }^{2}$ Rohit Garg ${ }^{1}$
}

${ }^{1}$ Department of Psychiatry, Government Medical College, Patiala, Punjab, India ${ }^{2}$ Department of Ophthalmology, Himalayan Institute of Medical Sciences, Swami Rama Himalayan University, Dehradun, Uttarakhand, India

Received: 06 March 2017 Accepted: 03 April 2017

*Correspondence to: Dr. Rajnish Raj, E-mail: drrajnish_raj@yahoo.com

Copyright: (C) the author(s), publisher and licensee Medip Academy. This is an openaccess article distributed under the terms of the Creative Commons Attribution NonCommercial License, which permits unrestricted noncommercial use, distribution, and reproduction in any medium, provided the original work is properly cited.

\begin{abstract}
Background: Few studies have reported the role of antidepressants as cataractogenic in humans.

Methods: It's a hospital based descriptive, cross-sectional study. 45 Patients were screened for antidepressant use and diminution of vision, 6 were dropped out. 39 patients with 78 eyes were finally enrolled. They were divided into two goups i.e., Group-I, with cataract $(\mathrm{N}=53)$ and Group-II, without cataract $(\mathrm{N}=25)$. Three clusters of antidepressants were assessed e.g., SSRI, SNRI and TCA with therapeutic dose (TD) and non-therapeutic dose (NTD) range. Psychiatric illness was diagnosed on DSM-5 and severity of depression on HAM-D. Best corrected visual acuity (BCVA) was converted from Snellen units to logarithm of minimal angle of resolution (log MAR) for statistical analysis. Cataract changes in eyes were seen on slit-lamp and classified on Lens opacities classification system-III (LOCS-III) criteria.
\end{abstract}

Results: A total 78 eyes of thirty-nine (39) patients were evaluated. Thirty $(38.46 \%)$ and forty-eight $(61.53 \%)$ eyes belonged to males and females, respectively. Mean age of males $(n=7)$ was $41.8 \pm 2.3$ years and females $(n=32)$ $40.2 \pm 1.0$ years. In Group-I, out of $(\mathrm{N}=53)$ eyes that developed cataract 33 were females $(62.26 \%)$ as compared to males $20(37.7 \%)$. Group -II, out of $(\mathrm{N}=25)$ eyes, females without cataract were $15(60 \%)$ as compared to males $10(40 \%)$. Therapeutic dose (TD) of antidepressants (AD) in Group-I had more cataract 37 $(69.81 \%)$ as compared to non-therapeutic dose (NTD) $16(30.1 \%)$. Most of the eyes with cataract $35(66.03 \%)$ had AD exposure of more than 1 year that was possibly associated with increased risk of cataract development (OR 2.10; $95 \%$ CI, 0.79-5.55). Amongst users of antidepressants, SSRI was associated with increased risk of cataract development (OR 2.4; 95\% CI, 0.72-7.94) with a female preponderance (OR 1.1; 95\% CI, 0.41-2.91). Maximum number of eyes $34(64.15 \%)$ that developed cataract had BCVA of $\geq 6 / 12$ and minimum of 2 $(3.77 \%)$ eyes had BCVA of $\leq 6 / 36$. LOCS-III revealed $38(71.69 \%)$ eyes $(71.69 \%)$ having peripheral cortical cataract and $15(28.30 \%)$ posterior subcapsular cataract.

Conclusions: There is a possible risk of association of cataract amongst user of antidepressants. The AD use of more than 1 year or longer had increased risk for development of cataract with a female preponderance. The highest risk was observed in the users of SSRI as compared to SNRI and TCA. Treatment exposure with antidepressant was longer for mild depression having more than two episodes.

Keywords: Diagnostic and statistical manual of mental health-5, Hamilton rating scale for depression, Lens opacities classification system-III, Selective serotonin reuptake inhibitor, Selective nor-epinephrine reuptake inhibitor, Tricyclic antidepressant

\section{INTRODUCTION}

Cataract is the leading cause of blindness worldwide. Oral and inhaled steroids increase risk of cataract. ${ }^{1}$ 
Recently, beta blockers are found to be cataractogenic. ${ }^{2}$ Beaver Dam Eye Study postulated the association of amitriptyline with increased risk of cortical cataract. ${ }^{3}$ The risk of cataract with newer generation antidepressants (AD) is unclear. Selective serotonin reuptake inhibitors (SSRIs) are the third most prescribed class of drug in the world and are associated with cataract gastrointestinal bleeding and fractures. ${ }^{4-8}$ Etminan and colleagues analyzed the data of 18,784 Quebec residents age more than $\geq 65$ years old with cataract and compared their health records with 187,840 aged-matched controls without cataract. ${ }^{9}$ They reported SSRIs having increased risk of cataract formation by about $15 \%$. Three antidepressants, which were commonly used in United States of America for depression led to possible increased risk of cataract development i.e., paroxetine had $23 \%$, venlafaxine $33 \%$ and fluvoxamine $39 \%$. Some of the antidepressants did not appear to be associated with cataract risk, possibly due to a chance factor.

Serotonin receptors (5-HT) are found in the lens of eyes. 5-HT represents a precursor for melatonin production in the pineal gland, crystalline lens and animal studies has shown that excess serotonin can make the lens opaque and lead to cataract formation. ${ }^{10}$ In the animal studies, serotonin neurotransmitter has shown to play a vital role in transparency of the lens and can lead in formation of cortical cataracts. ${ }^{5}$ The discovery of receptors for serotonin within the eye strongly suggests that 5-HT plays a functional role in the various ocular tissues. Serotonin inhibits the adenosine triphosphatase enzyme (ATPase) at the level of lens epithelium.

The physiological activity of this membrane enzyme represents a crucial point in maintenance of lens transparency. ${ }^{11}$ Evidence also suggests role of phosphoinositide cycle and its involvement in cellular signal transduction in the rabbit lens. ${ }^{12}$ Chidlow and coworkers have found very low levels in the lens of messenger ribonucleic acid (mRNA) for 5- $\mathrm{HT}_{1 \mathrm{~A}}$ and 5$\mathrm{HT}_{7}$ receptors, indicating that these receptors are expressed in epithelium. ${ }^{13}$ Seven distinct families of 5-HT receptors have been identified $\left(5-\mathrm{HT}_{1}\right.$ to $\left.5-\mathrm{HT}_{7}\right) \cdot{ }^{14}$ Similarly, detection of 5-HT receptor subtype mRNAs in numerous human ocular tissues..$^{15}$

The widespread use of SSRIs by psychiatrists and nonpsychiatrists poses a higher risk of cataract development and requires quarterly ophthalmological evaluation of patients for improving health care.

The study was to find out the role of AD as a risk factor and its association in terms of type, dose and duration of its use in development of cataract after controlling for the other associated biases e.g., elderly age, diabetes, hypertension, asthma, hypo or hyperthyroidism, hyperlipidemia, renal pathologies, any other systemic comorbidity, use of oral steroids and any other ocular ailments.

\section{METHODS}

It's an observational; hospital based; cross-sectional study conducted in tertiary Heath Care Centre of Government Medical College, Patiala. The study lasted for six months i.e., from June 2014 to December 2014. Written informed consent was obtained from all the subjects after they were given explanation of the nature of study. The study was conducted as per the Declaration of Helsinki and Good Clinical Practice Guidelines. The protocol had an approval of college ethical committee and confidentiality of the patient was maintained throughout the study period. The demographic profile of the patients was recorded. Prescription record of patient was retrieved, its strength, quantity, dosage and duration of treatment was extracted and recorded. During the study period, it was ensured that AD dosages are in the therapeutic range. Concomitant treatment with benzodiazepines and other hypnotics were permitted at the discretion of the investigator.

Any ophthalmological examination during the past one year or currently, complaining of blurring of vision or diminution of visual acuity was considered sufficient reason for further evaluation and assessment. Thus, the total of forty-five (45) patients were screened, Six (6) were dropped out as they withdrew consent. Finally, thirty-nine (39) patients with 78 eyes were enrolled. They were further divided into Group -I patients $(\mathrm{N}=53)$ with cataract and Group -II patients without cataract $(\mathrm{N}=25)$. The goal of the study was to test the null hypothesis (H0) of no association (e.g., $\mathrm{OR}=1$ ) between $\mathrm{AD}$ and development of cataract. However, if disapproved an alternate hypothesis (H1) stands validated for a possible association $(\mathrm{OR}<1$ or $\mathrm{OR}>1$ or $\mathrm{OR} \neq 1$ ) between antidepressant as a risk factor and an explanatory cause for the development of cataract i.e., an observed event.

\section{Inclusion criteria}

- All patients were below the age of 50 years who took antidepressants. They were further divided into three clusters/subclasses e.g., Selective serotonin reuptake inhibitor (SSRI), Selective nor-epinephrine reuptake inhibitor (SNRI) and Tricyclic antidepressant (TCA) e.g. sertraline, duloxetine and amitriptyline respectively.

- They were divided into two groups i.e., Group-I, with cataract and Group -II, without cataract; then based on the time duration, groups were further divided into $\mathrm{AD}$ exposure of $<1$ year and $>1$ year and also having psychiatric illness minimum of $>2$ episodes of major depressive disorder or more per year.

- Furthermore, according to their dosages they were divided into therapeutic dose (TD) and nontherapeutic dose (NTD) range. The TD of sertraline was $150-300 \mathrm{mg} /$ day, duloxetine $40-60 \mathrm{mg} /$ day and amitriptyline $150-225 \mathrm{mg} /$ day whereas less than the 
aforementioned therapeutic range was considered as NTD.

\section{Exclusion criteria}

- The patients having $\geq 50$ years of age.

- The patients with other co-morbidities like diabetes, hypertension, asthma, hyper or hypothyroidism, hyperlipidemia, oral steroid users and smokers.

- The patients with less than 2 episodes of depression.

- Patients with any other existing ocular pathology like glaucoma, uveitis or ocular trauma.

The psychiatric illness was diagnosed by the consultant Psychiatrist on Diagnostic and Statistical Manual of Mental Health-5 (DSM-5) and severity of depression on Hamilton Rating Scale for Depression (HAM-D). ${ }^{16,17}$ The degree of severity for mild, moderate and severe depression was in the range 10-13, 14-17 and >17 respectively. The presence or absence of cataract was assessed and diagnosed by the consultant Ophthalmologist. Battery of ophthalmological examinations were conducted e.g., refraction, intraocular pressure and slit-lamp examination of anterior segment and fundus examination. Best corrected visual acuity (BCVA) was converted from Snellen units to logarithm of minimal angle of resolution (logMAR) for statistical analysis. Cataractous changes were assessed on slit-lamp examination after full dilatation and on retroillumination. To classify cataract, Lens opacities classification system-III (LOCS-III) was used. ${ }^{18}$

\section{Statistical analysis}

Data were initially entered into an excel spreadsheet and then transferred to SPSS software (Statistical Package for
Social Sciences, version 20, SPSS Inc, Chicago, IL). The result was expressed as mean \pm standard deviation. Independent t-test was used to compare the age of patient between two groups i.e., Group I and Group II, respectively based on presence and absence of cataract. Qualitative data were expressed as frequency and percentage. Fisher's exact test was used to check the association between $\mathrm{AD}$ as a risk factor e.g., their type, dosage, duration of drug intake and development of cataract. Univariate and multivariate logistic regression analysis was used to estimate odds ratio (OR) of developing cataract in different categories. To summarize their relationships, estimated OR with $95 \%$ of confidence intervals (CI) was used after adjusting for gender and other confounding variables e.g., types, duration and dosage of AD. Depression was diagnosed on DSM-5 and their severity on HAM-D. Mann-Whitney test was used to compare the BCVA (logMAR) values of patient who developed cataract with $\mathrm{AD}$ having duration of exposure $<1$ year and $>1$ year. The P-value less than $(<0.05)$ was considered statistically significant.

\section{RESULTS}

Table 1: Showing distribution of patients in different types of antidepressants.

\begin{tabular}{|lll|}
\hline Drug groups & No. of eyes involved (N) & $\%$ (Eyes) \\
\hline SSRI & 37 & 47.43 \\
\hline SNRI & 24 & 30.76 \\
\hline TCA's & 17 & 21.79 \\
\hline Total & 78 & 100.00 \\
\hline
\end{tabular}

SSRI Selective serotonin reuptake inhibitor, SNRI Selective nor-epinephrine reuptake inhibitor, TCA's Tricyclic antidepressants

Table 2: Association between development of cataract and various risk factors among patients using various antidepressants.

\begin{tabular}{|c|c|c|c|c|c|c|}
\hline & & $\begin{array}{l}\text { Group-I } \\
\text { (with cataract) } \\
N=53(\%)\end{array}$ & $\begin{array}{l}\text { Group-II } \\
\text { (without cataract) } \\
\mathrm{N}=\mathbf{2 5}(\%)\end{array}$ & $\begin{array}{l}\text { p } \\
\text { value* }\end{array}$ & $\begin{array}{l}\text { Odd's ratio } \\
\text { (95\% CI) }\end{array}$ & $\begin{array}{l}\text { Adjusted Odd's } \\
\text { ratio } \\
(95 \% \text { CI })\end{array}$ \\
\hline \multirow{2}{*}{ Gender } & Male & $20(37.7)$ & $10(40)$ & \multirow{2}{*}{1.0} & 1 & 1 \\
\hline & Female & $33(62.2)$ & $15(60)$ & & $1.1(0.41-2.91)$ & $1.08(0.31-3.71)$ \\
\hline \multirow{3}{*}{ Drug groups } & SSRI & $27(50.9)$ & $10(40)$ & \multirow{3}{*}{0.3} & $2.4(0.72-7.94)$ & $1.98(0.49-7.94)$ \\
\hline & SNRI & $17(32.0)$ & $7(28)$ & & $2.15(0.59-7.89)$ & $1.85(0.44,7.79)$ \\
\hline & TCA's & $9(16.9)$ & $8(32)$ & & 1 & 1 \\
\hline \multirow{2}{*}{ Dose } & TD & $37(69.8)$ & $14(56)$ & \multirow{2}{*}{0.3} & $1.81(0.67-4.85)$ & $1.07(0.31-3.72)$ \\
\hline & NTD & $16(30.1)$ & $11(44)$ & & 1 & 1 \\
\hline \multirow{2}{*}{$\begin{array}{l}\text { Duration of } \\
\text { drug use }\end{array}$} & $<1$ year & $18(33.9)$ & $13(52)$ & \multirow{2}{*}{0.1} & 1 & 1 \\
\hline & $>1$ year & $35(66.0)$ & $12(48)$ & & $2.10(0.79-5.55)$ & $1.53(0.50-4.69)$ \\
\hline \multirow{2}{*}{$\begin{array}{l}\text { Episodes of } \\
\text { depression per } \\
\text { year }\end{array}$} & 2 & $38(71.6)$ & $16(64)$ & \multirow{2}{*}{0.6} & 1 & 1 \\
\hline & 3 & $15(28.3)$ & $9(36)$ & & $0.70(0.25-1.93)$ & $0.58(0.19-1.78)$ \\
\hline \multirow{2}{*}{ HAM D Score } & Mild & $38(71.6)$ & $12(48)$ & \multirow{2}{*}{0.04} & 1 & 1 \\
\hline & Moderate & $15(28.3)$ & $13(52)$ & & $2.74(1.02-7.35)$ & $2.54(0.77-8.28)$ \\
\hline
\end{tabular}

$\mathrm{P}$ value*calculated by Fisher exact test, TD-Therapeutic dose, CI-Confidence interval, NTD-Non-therapeutic dose, TCA-Tricyclic antidepressant, SSRI-Selective serotonin reuptake inhibitor, SNRI-Selective nor-epinephrine reuptake inhibitor, HAM-D score Hamilton Rating Scale for Depression scores for mild, moderate and severe depression were in the range of 10-13, 14-17 and >17 respectively. 
A total 78 eyes of thirty-nine (39) patients were included for the study. Thirty $(38.46 \%)$ and forty-eight $(61.53 \%)$ eyes belonged to males and females, respectively.

Mean age of males $(n=7)$ was $41.8 \pm 2.3$ years and females $(n=32) 40.2 \pm 1.0$ years. Minimum and maximum age of the patient was 22 and 49 years, respectively. Patients on AD were clustered into three groups e.g. SSRI, SNRI and TCA's. Their pattern of distribution amongst patients is shown in (Table 1).

In the primary analysis, females had higher risk of cataract development as compared to males. In Group-I, $(\mathrm{N}=53)$ eyes with cataract 33 were of females $(62.26 \%)$ as compared to males $20(37.7 \%)$. Group-II, $(\mathrm{N}=25)$ eyes females without cataract were $15(60 \%)$ as compared to males $10(40 \%)$. Group-I with therapeutic dose (TD) of antidepressants (AD) had more cataract $37(69.81 \%)$ as compared to non-therapeutic dose (NTD) 16 (30.1\%). Most of the cataractous eyes $35(66.03 \%)$ had AD exposure of more than 1 year that was associated with increased risk of cataract development (OR 2.10; 95\% CI, 0.79-5.55). Similarly, amongst users of AD, SSRI was associated with increased risk of cataract development (OR 2.4; 95\% CI, 0.72-7.94) with a female preponderance (OR 1.1; 95\% CI, 0.41-2.91). Patient with mild degree of depression on HAM-D score showed statistically significant development of cataract $(\mathrm{P}=0.04)$ (Table 2).

Table 3: Best corrected visual acuity (BCVA) converted to logarithm of minimal angle of resolution (logMAR) in patients who developed cataract with various antidepressants.

\begin{tabular}{|c|c|c|c|c|c|}
\hline & $\begin{array}{l}\text { Duration of antidepressant } \\
\text { exposure }\end{array}$ & $\mathbf{N}$ & Mean \pm SD & $\begin{array}{l}\text { Range } \\
\text { (maximum-minimum) }\end{array}$ & P value \\
\hline \multirow{2}{*}{$\begin{array}{l}\text { BCVA }(\log \text { MAR) } \\
\text { in patients with cataract }\end{array}$} & $<1$ Year & 18 & $0.34 \pm 0.11$ & $0.7-0.2$ & \multirow{2}{*}{0.07} \\
\hline & $>1$ year & 35 & $0.40 \pm 0.14$ & $0.7-0.3$ & \\
\hline
\end{tabular}

$\mathrm{P}$ value\# calculated by Mann-Whitney test, BCVA-Best corrected visual acuity, logMAR-Logarithm of minimal angle of resolution.

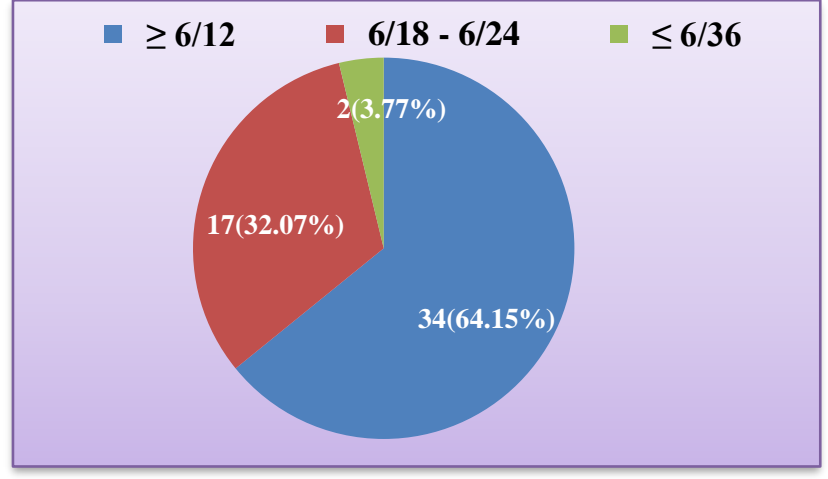

Figure 1: The distribution of BCVA (Snellen acuity) among the eyes which developed cataract.

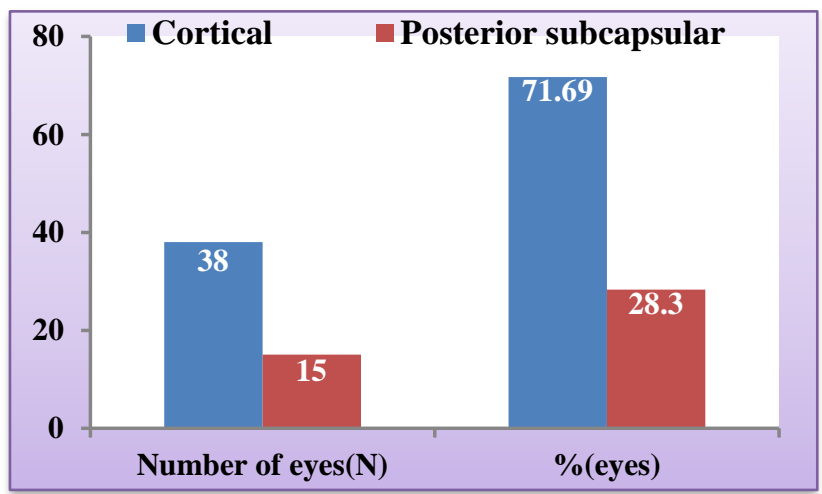

Figure 2: The distribution of different types of cataract.
In Group-I, the comparison amongst risk of AD users of duration $<1$ year and $>1$ year and development of cataract showed mean value for BCVA ( $\log$ MAR) was $0.34 \pm 0.11$ and $0.40 \pm 0.14$ with $\mathrm{p}$-value of 0.07 , which was nonsignificant (Table 3 ).

Out of the 53 eyes of the patients, maximum of 34 $(64.15 \%)$ had cataract with BCVA of $\geq 6 / 12$ as compare to minimum of $2(3.77 \%)$ having BCVA of $\leq 6 / 36$ (Figure 1 ). Lens opacities classification system-III (LOCS-III) revealed $38(71.69 \%)$ peripheral cortical cataract and 15 (28.30\%) posterior sub-capsular cataract. (Figure 2, $3 \mathrm{a}, \mathrm{b}$ and $4 \mathrm{a}, \mathrm{b})$.

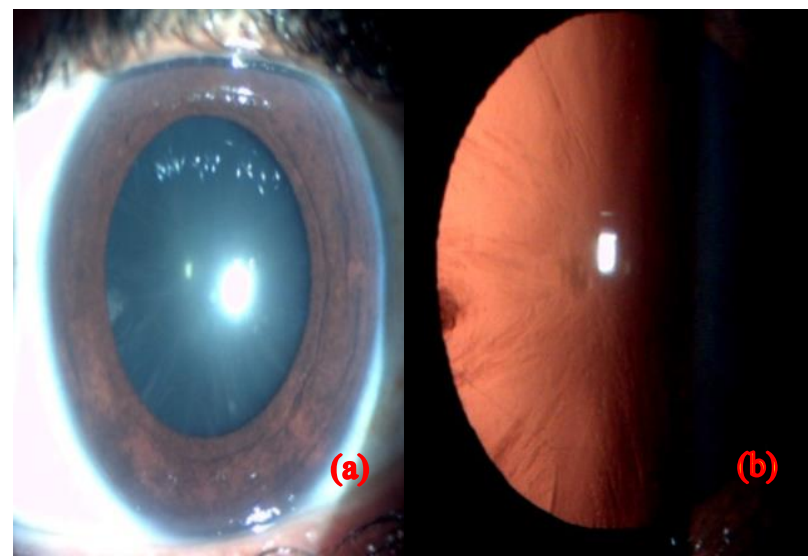

Figure 3 (a, b): Peripheral cortical cataract on diffuse illumination and retro-illumination. 


\section{DISCUSSION}

The present study showed that $\mathrm{AD}$ was associated with increased risk of cataract at a relatively younger age i.e. $<50$ years, which cannot be attributed to a bias of senile cataract. Females were at higher risk of cataract as compared to males. SSRIs were strongly associated with cataract as compared to other classes of AD e.g., SNRI and TCA. Drug intake of $>1$ year was associated with higher rate of cataract formation as compared to $<1$ year with (OR 2.10, 95\% CI 0.79-5.55, $\mathrm{P}=0.14$ ), which was statistically non-significant. These findings were in agreement with the study by Eries and colleagues that found use of SSRI for 1 or more years associated with an increased risk of cataract (OR 1.36, 95\% CI 1.23-1.51; $\mathrm{P}<0.001)$. The association between SSRI use and incident of cataract surgery was significant for men (OR 1.34, 95\% CI 1.12-1.61) and women (OR 1.37, 95\% CI 1.221.55). It remained significant after adjusting for risk factors of cataract formation e.g., diabetes mellitus and steroid use $(\mathrm{P}<0.001){ }^{19}$

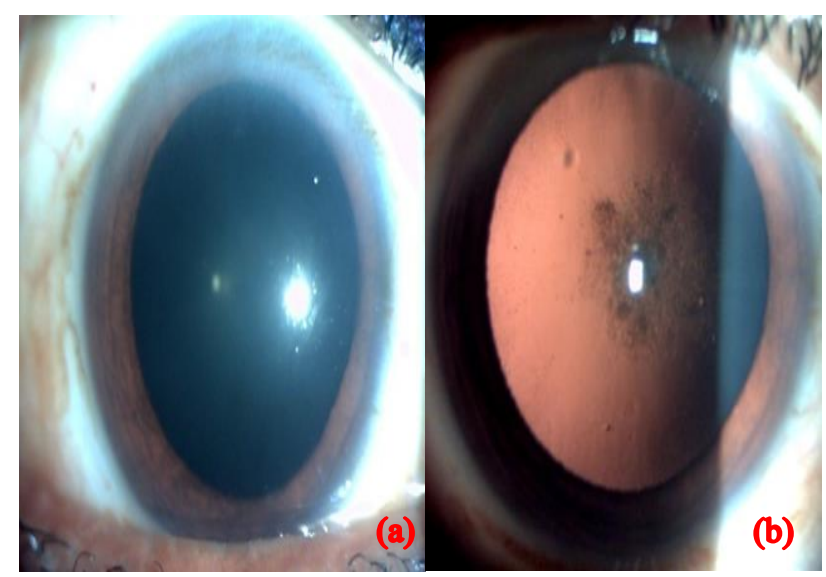

Figure 4 (a, b): Posterior sub-capsular cataract on diffuse illumination and retro-illumination.

Etminan et al., 2010 studied 200,000 residents with age of 65 years and above and reported a possible association between current use to SSRIs i.e., $15 \%$ greater relative risk (RR) of cataracts (95\% CI 1.08 to 1.23 ) than nonusers after adjusting for confounders e.g., high blood pressure, other medication use and gender etc. The antidepressant drugs e.g., fluvoxamine, venlafaxine and paroxetine had highest risk of cataracts whereas citalopram, fluoxetine and sertraline did not show statistically significant increase. ${ }^{9}$ In their study, the average time duration to diagnosis of cataract while patients were on SSRI therapy was 656 days. However, the incidence of community diagnosed cataracts had (adjusted rate ratio $1.06,95 \%$ CI 0.97 to 1.17 ) with past SSRI use. Amongst SSRIs, the past use of sertraline had $19 \%$ elevated risk of cataract (95\% of CI $1 \%$ to $41 \%$ ). Any current use of sertraline with cataract diagnosis showed no significant effect on cataract risk (adjusted RR $1.06,95 \%$ CI 0.92 to 1.22 ).
In the present study, $27(50.9 \%)$ eyes were exposed to SSRI (sertraline) use and developed cataract, which was statistically non-significantly (OR 2.4, 95\% CI 0.72-7.94, $\mathrm{P}=0.3$ ). The findings were consistent as reported earlier by Etminan and colleagues. ${ }^{9}$ This may suggest importance of selective serotonin receptors in the formation of cataracts. Catecholamines such as norepinephrine, having catarogenic properties, and premarketing clinical trials suggest an association between cataracts and venlafaxine ${ }^{6}$ whether, it's a cataractogenic effect of SSRI or a class effect or limited to specific agents must be investigated in future.

Serotonins have been shown to play a crucial role in lens transparency. ${ }^{12}$ Animal studies have revealed the role of serotonin in different areas of eye. ${ }^{5} \mathrm{~A}$ wide variety of serotonin receptors have been identified in the lens of animal models. ${ }^{10,13}$ Cataractogenic potential of serotonin was observed in rat model with lens opacification and formation of cortical cataracts after injection of $1 \%$ solution of serotonin. ${ }^{5}$ In the current study, maximum number of eyes had peripheral cortical cataract, which was visually non-significant but in concordance with the observation by Boerrigter et al. ${ }^{5}$ One of the postulates for lens opacification is serotonin interfering with lens metabolism, which compromises ciliary body and anterior chamber. The presence of $\beta$ receptors in lens and role of cataractogenic properties of catecholamines ${ }^{6}$ should be further investigated.

In the present study after adjusting for gender and other potential confounding variables e.g., types of $\mathrm{AD}$, duration of drug intake, dosage, episodes of depression and severity of depression, the use of SSRIs for $>1$ year was associated with increased risk of $35(66 \%)$ cataract development (OR 2.10, 95\% CI 0.79-5.55, $\mathrm{P}=0.1$ ) as compared to the duration of $<1$ year but statistically nonsignificant. The dosage of $\mathrm{AD}$ was a possible risk factor for the development of cataract. The TD showed increase in the development of cataract $37(69.8 \%)$ eyes (OR 1.81, $95 \%$ of CI $0.67-4.85)$ as compared to non-therapeutic dose (NTD) $16(30.1 \%)$. The BCVA for maximum number of eyes $34(64.15 \%)$ was $\geq 6 / 12$, which required no cataract surgery.

\section{Limitations}

- It's a prescription based study to link the prescription data with psychiatric and ophthalmological diagnoses.

- This study assessed cataract formation rather than cataract surgery and an effort to find an association between the $\mathrm{AD}$ and development of cataract.

- The other risk factors that may confound the results were controlled; they were non-significant but statistically or clinically relevant in assessment for development of cataract.

- The odd ratios were calculated. The wide confidence interval showed that results are not much reliable due to small sample size 


\section{Implication}

The study highlights the role of SSRI as a risk factor for development of cataract in humans.

\section{CONCLUSION}

There is a higher risk of association with cataract formation amongst users of SSRI than SNRI and TCA. SSRI users having exposure more than 1 year or longer for mild degree of depression developed visually nonsignificant peripheral cortical cataract to significant cataract with a female preponderance. Thus, it is recommended that clinicians using $\mathrm{AD}$ of longer duration for eligible patients should have periodic follow-up by ophthalmologists to rule out incipient cataract. A larger naturalistic cohort and survival analysis studies are required to establish role of $\mathrm{AD}$ having cataractogenic potential before generalizing these results.

\section{ACKNOWLEDGEMENTS}

Authors would like to express their sincere thanks to $\mathrm{Mr}$ Hem Chandra Sati, Statistical Assistant of All India Institute of Medical Sciences, New Delhi for his immense help in the Biostatistics

Funding: No funding sources Conflict of interest: None declared

Ethical approval: The study was approved by the Institutional Ethics Committee

\section{REFERENCES}

1. Ernst P, Baltzan M, Deschenes J, Suissa S. Low-dose inhaled and nasal corticosteroid use and the risk of cataracts. Eur Respir J. 2006;27:1168-74.

2. Kanthan GL, Wang JJ, Rochtchina E, Mitchell P. Use of antihypertensive medications and topical betablockers and long- term incidence of cataract and cataract surgery. Br J Ophthalmol. 2009;93:1210-14.

3. Klein BE, Klein R, Lee KE, Danforth LG. Drug use and five-year incidence of age-related cataracts: the beaver dam eye study. Ophthalmol. 2001;108:16704.

4. Ludwig J, Marcotte DE, Norberg K. Anti-depressants and suicide. J Health Econ. 2009;28:659-76.

5. Boerrigter RM, Sietsema JV, Kema IP. Serotonin (5HT) and the rat's eye: some pilot studies. Doc Ophthalmol. 1992;82(1-2):141-50.

6. Weinstock M, Scott JD. Effect of various agents on drug induced opacities of the lens. Exp Eye Res.1967;6:368-75.

7. Van Walraven C, Mamdani MM, Wells PS, Williams JI. Inhibition of serotonin reuptake by antidepressants and upper gastrointestinal bleeding in elderly patients: retrospective cohort study. BMJ. 2001;323:655-8.

8. Ginzburg R, Rosero E. Risk of fractures with selective serotonin-reuptake inhibitors or tricyclic antidepressants. Ann Pharmacother 2009; 43: 98-103.

9. Etminan M, Mikelberg FS, Brophy JM. Selective serotonin reuptake inhibitors and the risk of cataracts. Ophthalmology. 2010;117:1251-5.

10. Costagliola C, Parmeggiani F, Sebastiani A. SSRIs and intraocular pressure modifications: evidence, therapeutic implications and possible mechanisms. CNS Drugs. 2004;18(8):475-84.

11. Candia OA, Lanzetta PA, Alvarez LJ, Gaines W. Inhibition of ionic transport and ATPase activities by serotonin analogues in the isolated toad lens. Biochem Biophys Acta. 1980;602:389-400.

12. Vivekanandan S, Lou MF. Evidence for the presence of phosphoinositide cycle and its involvement in cellular signal transduction in the rabbit lens. Curr Eye Res. 1989;8:101-12.

13. Chidlow G, Le Corre S, Osborne NN. Localization of 5-hydroxytryptamine-1A and 5-hydroxytryptamine-7 receptors in rabbit ocular and brain tissues. Neuroscience. 1998;87:675-89.

14. Hoyer D, Hannon JP, Martin GR. Molecular, pharmacological and functional diversity of 5-HT receptors. Pharmacol Biochem Behav. 2002;71:53354.

15. Sharif NA, Senchyna M. Serotonin receptor subtype mRNA expression in human ocular tissues, determined by RT-PCR. Mol Vision. 2006;12:10407.

16. American Psychiatric Association. (DSM-5) Diagnostic and statistical manual of mental disorders (5th ed.) Arlington, VA: American Psychiatric Publishing. 2013:155-88.

17. Hamilton M. Development of a rating scale for primary depressive illness. Br J Soc Clin Psychol. 1967;6(4):278-96.

18. Chylack LT, Wolfe JK, Singer DM et al. Lens Opacities Classification System III. The Longitudinal Study of Cataract Study Group. Arch Ophthalmol. 1993;111:831-6.

19. Erie JC, et al. Selective serotonin reuptake inhibitor use and increased risk of cataract surgery: a population-based, case-control study. Am J Ophthalmology. 2014;158:192-7.

Cite this article as: Raj R, Raj A, Garg R.

Antidepressants-a possibly risk factor for cataract development: a cross-sectional study. Int J Basic Clin Pharmacol 2017;6:1095-100. 